\title{
EDITORIAL
}

\section{Reference patterns and crystal structures of pharmaceuticals}

The September 2015 edition of Powder Diffraction journal (PDJ) contains a number of contributions dealing with important active pharmaceutical ingredients (APIs) and related compounds. These appear in Powder Diffraction as Technical Articles, Rapid Communications or Data Reports, depending on the level of information in the literature and the Powder Diffraction File (PDF). These papers provide state-of-the-art $\mathrm{X}$-ray powder diffraction data and crystal structure analysis along with exceptional quality X-ray diffraction reference powder patterns. They result from collaboration between The International Centre for Diffraction Data ${ }^{\circledR}$, Illinois Institute of Technology, Poly Crystallography Inc., and Argonne National Laboratory (ANL). Their goal is to report the crystal structures and reference patterns for many of the largest dollar volume APIs whose crystal structures are currently unknown. In their Technical Article papers the crystal structure analyses were determined from the exceptional high quality synchrotron powder diffraction data collected at ANL using constraints in Rietveld refinement. The structures were then optimized using computationally intensive density functional theory geometry optimization. While this optimization may introduce small changes in the atomic coordinates, they consistently yield better torsion angles, bond distances, and bond angles.

Within this volume of Powder Diffraction there are also a series of papers from the College of Chemical Engineering, Sichuan University, Chengdu, China that have also reported high quality reference patterns of APIs. The continuation of contributions of high accuracy should contribute to the ongoing worldwide efforts to improve knowledge and characterization of active pharmaceuticals. 